\title{
The Design and Testing of a LiDAR Platform for a UAV for Heritage Mapping
}

\author{
Li Zhe ${ }^{\mathrm{a} *}$, Yan Yu ${ }^{\mathrm{a}}$, Jing Yabing ${ }^{\mathrm{b}}$, Zhao ShuGuang ${ }^{\mathrm{c}}$ \\ ${ }^{a}$ National Key Lab of Ancient Building Surveying; School of Architecture, Tianjin University, Tian Jin,300072, China \\ lee_uav@tju.edu.cn \\ ${ }^{\mathrm{b}}$ Technical Center of Vibration and Noise, Institute of Internal Combustion Engine, Tianjin University, Tian Jin, 300072, China \\ robin@tju.edu.cn \\ c Tianjin Aurora UAV Technology Co., Ltd., Tian Jin, 300201, China uav_tuna@163.com
}

Commission I, ICWG I/Vb

KEY WORDS: architectural relics, laser scanning, LiDAR, vibration interference, VTOL aircraft

\begin{abstract}
:
In the discovering, identifying and mapping work of heritage objects in forest or desert areas, LiDAR ensures work efficiency and can provide the most complete and accurate 3D data. In the field of heritage documentation in China, the integration of LiDAR and small UAV is highly desirable. However, due to issues on the vibration of flying platform, load capacity, safety and other factors, not all UAVs can be used as LiDAR carriers. Therefore, the selection and design of suitable UAVs are very important. Little research has been done in this area and related experiments, complete test data and clear conclusions are hard to find. After long-term selection, design, trial-manufacturing and testing, the authors compare the vibration, capacity, reliability, stability of many UAV types, and finally develop two UAV platforms which are most suitable for carrying LiDAR for heritage mapping projects.
\end{abstract}

\section{INTRODUCTION}

Ancient architectural relics in southern China are usually covered by verdant trees, making it difficult to collect accurate surface data by photogrammetry methods. In northwest China, vegetation is sparse, but relics usually have sharp light and shade contrast and lack natural texture, resulting in point clouds generated by photogrammetry incomplete. Therefore, photogrammetry has limited application and UAV based LiDAR use in the mapping of small sites containing architectural relics in the southern woodlands and the northwest desert would offer several benefits.

Architectural heritages and relics are usually found in areas of complex terrains, small in dimensions but demanding high measurement accuracy for useful results. Rotor VTOL UAVs are considered the most suitable type for the data collection of such relics. The UAV family has many members, each varying in power, structure and performance. Not all can be used as LiDAR platforms. Based on the following factors, the authors, after many experiments, propose two desirable UAVs for LiDAR. Factors to be considered when utilizing UAVs as LiDAR platforms and discussed further in this paper, are:

1. Vibration -- Vibration interferes with the angular accuracy of the LiDAR's IMU, causing a data error. Heritage mapping requires high-precision data and is hence sensitive to platform vibration.

2. Safety -- LiDAR is expensive and fragile and UAVs have a high failure rate. Therefore, a protection cage is needed to minimize the possibility of LiDAR damage.

3. Capacity \& Duration - compared with a camera, LiDAR is heavier and slower in data collection. The load capacity of the carrier should be $5-20 \mathrm{~kg}$ and flight duration no less than 20-60 minutes.
4. flight quality, maintenance complexity, the blocking of the laser beam by UAV body -- factors that must be considered while designing a LiDAR platform.

\section{LASER SCANNING AND VIBRATION INTERFERENCE}

Airborne laser scanning has both internal and external errors. The former is inherently, related to the performance index of the laser head, IMU, GPS and other components as well as the calibrated quality of these combined components. There has been considerable research on internal errors, and this paper mainly focuses on the source and elimination of external errors.

External factors that affect laser scanning accuracy include: "atmospheric turbulence, platform stability, air pressure and humidity ... Furthermore, to the best of the author's knowledge, there are no existing researches concerning the positioning errors caused by the instability of the platform that a LiDAR system installed. Such an instability can be caused by either the atmospheric disturbance or the vibration from the platform engine. "[1]. In heritage mapping, the UAV mainly hedgehops and scans at close range, and therefore interference of the laser beam transmission by such factors as air pressure, airflow, humidity changes and other factors can be negligible. However, with rotary inertia generated by the motors of the UAV as well as from simple vibration reduction or isolation parts, flutter and vibration caused by these components is easily transmitted to the body and LiDAR. This leads to greater vibration interference, compared with larger more stable manned aerial vehicle. As architectural heritage mapping is more demanding than topographic mapping, ones priority is to reduce UAV vibration interference. "The impact of platform vibration on the final data can be summarized as: Platform vibration does

\footnotetext{
* Corresponding author
} 
impact on the positioning accuracy of a LiDAR system Measurements should be adopted to reduce platform vibration in order to get much higher accuracy of the result data" [1 ].

\section{CAUSE ANALYSIS, QUANTIFICATION AND ELIMINATION OF VIBRATION}

For fixed-wing, manned aircraft, vibration is caused not only by the engine, but also by wind flow turbulence passing over the body, pod bay and rudder surface control [1]. VTOL UAVs usually fly at low speed, hence turbulence vibration generated by body shape can be negligible- For this platform, vibration mainly comes from the engine, rotor and gears.

Vibration is a very complicated factor, which is especially true of such moving platforms as UAVs. Vibration originating from its mechanical components has a multilevel and interconnected relationship. Therefore it is difficult to identify, quantify and reduce vibration interference merely by mathematical calculation. The identification and quantification of vibration relies on tests and the elimination of vibration relies on data analysis and improvement. "To analyze vibration analytically is almost impossible due to its complexity ... This indicates that errors caused by vibration are much more complicated so that traditional methods are hard to remove them (Kager H, 2004)... We get another conclusion that causes and effects of the platform vibration to LiDAR system are complex, so no simple plant or surface equations can fit the error distribution well. "[1] Since interference cannot be resolved through algorithm optimization of the IMU itself, one should consider reducing the vibration of the carrier itself. The primary task is not just to install various dampers for LiDAR, but to consider the selection, design and testing of the entire UAV platform and systems.

Studies have shown that dampers are not very effective, especially when it comes to the UAV platform. "Flexible supports, namely, dampers, are largely used in the installation of IMU. However, when the elastic center does not coincide with the center of mass, line vibration and angular vibration is likely to generate coupled vibration ... since devices have low tolerance for torsional vibration, large eccentricity should be avoided but is actually unavoidable for a variety of reasons. Thus, the most prudent approach is to avoid using long and narrow structures so that their influence can be reduced to the minimum. "[7] Due to the arrangement of laser head, IMU and GPS, LiDAR systems are usually long and narrow in shape, thus vibration is not easily eliminated. Furthermore, UAV vibration interference has multi-band frequency and is of multiamplitude nature. One damper is only applicable in a certain frequency band. Therefore, a vibration reduction mechanism made up of multi-level dampers is needed to deal with vibration of high and low frequency respectively, which will significantly increase the weight (reference [2]) and reduce the payload of the UAV. For example, an experiment with a LiDAR system installed on a piston helicopter (max weight $100 \mathrm{~kg}$ ) showed that a composite spring damping mechanism (Figure 1) failed to completely eliminate vibration at various frequencies from 15 $\mathrm{Hz}$ (the main rotor) to $200 \mathrm{~Hz}$ (the engine). It even induced extra flutter (low-frequency torsional vibration coupling) when the aircraft increased or decreased its speed. Therefore, one should think of ways to reduce vibration originating from the UAV platform itself. Through the selection of key components, integrated design and trial manufacturing, one is able to compare vibrations on different platforms and in different working conditions in order to find an optimal UAV (helicopter or drone) to carry a LiDAR system.

\section{THE SELECTION, DESIGN AND TESTING OF HELICOPTER}

Helicopters are principle members of the VTOL family. This paper analyses the optimal unmanned helicopter platform for LiDAR from three aspects, i.e, the engine, rotor and their configuration.

\subsection{Selection of power unit}

Piston engine propulsion is not suitable for a LiDAR carrier because it's explosive stroke is an undesirable source giving rise to strong vibrations of more frequencies which are transmitted to the helicopter's main structure.

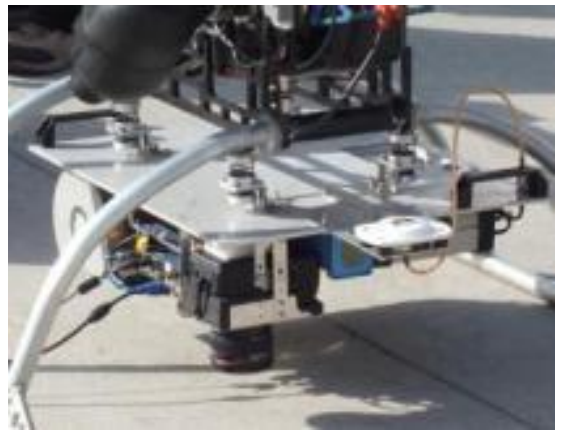

Figure 1 Damping parts of Lidar on a 100kg gasoline helicopter



Figure 2 Vibration frequency at the front part of a $15 \mathrm{~kg}$ gasoline helicopter

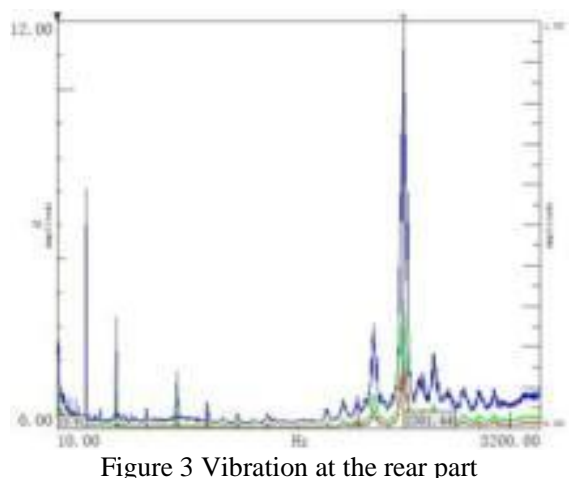

Figure 2 shows the vibrational frequency at a measuring point at the front of a $15 \mathrm{~kg}$ gasoline-powered helicopter in a hovering state. Figure 3 shows the vibrational frequency at a measuring point at the rear of the same helicopter under the same working conditions. As can be seen, vibration in the front part is mainly engine vibration, peaking at $8 \mathrm{G}, 195.79 \mathrm{~Hz}$, with the same rotation speed as that of a gasoline engine (11747 r/min), and 
the frequency doubling to be $398.48 \mathrm{~Hz}$, a secondary peak is derived from the reciprocating motion of the piston. Vibration at the rear part of the helicopter reads $12 \mathrm{G}, 2301.44 \mathrm{~Hz}$, which is of high strength and high frequency. Since there are no such high-speed rotating parts on the helicopter, the accumulated vibration is generated by the engine. In contrast, the lowfrequency vibration (peak $1.7 \mathrm{G}$ ) produced by the main rotor $(1500 \mathrm{r} / \mathrm{min})$ and the tail rotor $(8250 \mathrm{r} / \mathrm{min})$ can be negligible. Therefore, a piston engine is the primary source of a helicopter's vibration, constituting a major interference for a LiDAR system.

A multi-cylinder combination is primarily used for reducing the vibration of internal combustion engines. Some special internal combustion engines such as the rotary engine have also been developed to reduce stroke vibration. Despite these configurations, it is not possible to avoid vibration caused by the explosion stroke. Moreover, a rotary engine is difficult to manufacture and maintain and is thus not widely used. This has lead the authors to design a helicopter with a turbine-shaftengine and to analyse its vibration, in the hope of completely eliminating stroke interference.

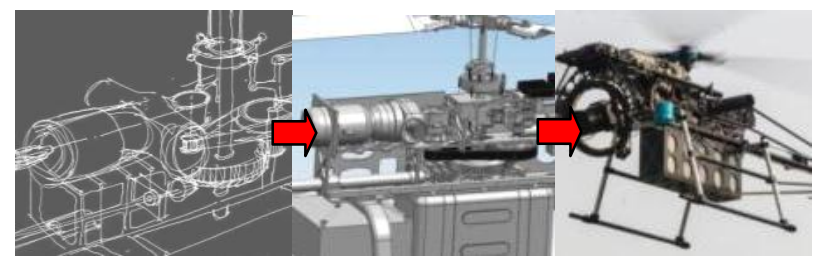

Figure 4 R\&D of a turbine-shaft UAV

This helicopter was is equipped with an $8 \mathrm{KW}$ turbine-shaft engine, with maximum takeoff weight being $40 \mathrm{~kg}$. It's single main rotor has three blades with diameter of $2.6 \mathrm{~m}$. The development process included design, mechanical drawing, parts fabrication, assembly and commissioning (Figure 4). Two models (A and $\mathrm{B}$ ) were made for controlled trials. After a trial flight, vibrations of the two models at different parts, in different working conditions were measured. The vibrations of the two models were compared with those of gasoline engine powered helicopters to verify the validity of power selection and data repeatability.

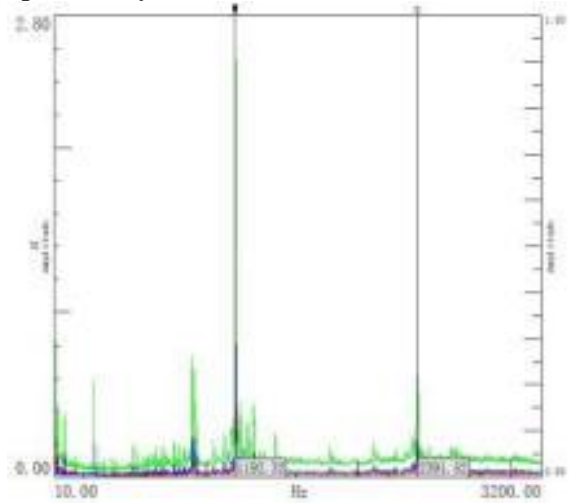

Figure5 Body vibration frequency of turbine shaft helicopter (main rotor $800 \mathrm{r} / \mathrm{min}$ )

Helicopter A was not carrying a LiDAR. In Figure 5, when its main rotor ran at $800 \mathrm{r} / \mathrm{min}$, the primary turbine of the turbineshaft engine running at $143,491.2 \mathrm{r} / \mathrm{min}$ generated a vibration of only $0.65 \mathrm{G}$ at $2391.52 \mathrm{~Hz}$; and the secondary turbine (power output turbine) running at $71,539.2 \mathrm{r} / \mathrm{min}$ produced a peak vibration of $2.8 \mathrm{G}$ at $1192.32 \mathrm{~Hz}$, far less than that of a gasoline helicopter, even though the turbine helicopter is much bigger. Helicopter B was carrying a LiDAR, and with the main rotor running at over $1000 \mathrm{r} / \mathrm{min}$, the unidirectional peak vibration of the secondary turbine can approaches 9G (Figure 6). A comparative analysis of the two figures showed that the turbineshaft engine induced very little vibration of other frequencies and that peak vibration at very high frequency section on $\mathrm{Y}$ axis (left and right side of body) was due to the installation of a mounting rack at the center of the engine near the primary turbine of Helicopter B. Vibration was significantly reduced after the removal of this mounting rack. Peak vibrations at low frequencies were caused by the rotation of gears, tail rotor and main rotor (Figure 7).

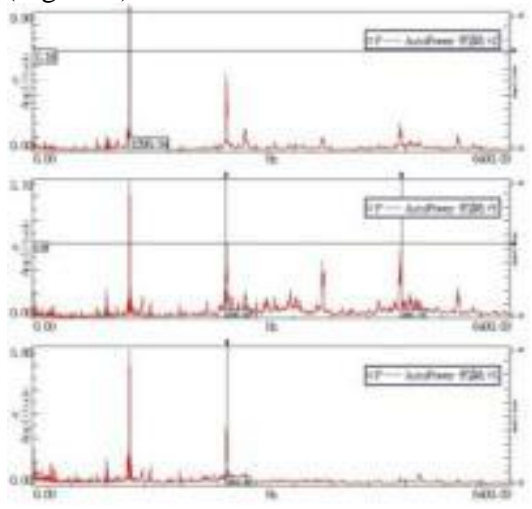

Figure 6 Body vibration (1021 r/min)

As can be seen from data analysis, vibration intensity of the turbine-shaft engine (especially the secondary turbine) mainly depends on the dynamic balancing. The smaller a turbine-shaft engine, the higher its rotation speed and the more difficulty in balancing the system. This engine is the smallest model of the turbine-shaft engine family. The larger the engine, the more easily they are manufactured, and the greater the choice of available engines. In theory, the fuel efficiency of a turbineshaft engine increases with its size so that for a larger LiDAR system, a turbine-shaft helicopter is a wise choice. Compared with a piston engine, a turbine significantly reduces the vibration of the helicopter body. Its low vibration interference makes it a good power source for a LiDAR helicopter.

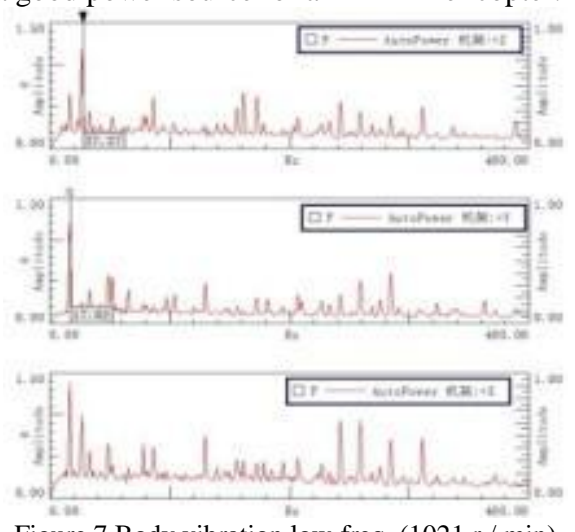

Figure 7 Body vibration low-freq. (1021 r/min)

Further, a turbine-shaft engine is superior in that it has a high thrust-to-weight ratio and longer hovering time. For example, the weight of the engine used in this test was $2.5 \mathrm{~kg}$, but the helicopter's maximum take-off weight is $40 \mathrm{~kg}$, a ratio of $1: 16$. The helicopter would be capable of arrying a $10 \mathrm{~kg}$ LiDAR system. In standard working conditions (i.e., at sea level altitude and a normal temperature of $25^{\circ} \mathrm{C}$ ) and with a take-off weight of $35 \mathrm{~kg}$ (including the $5 \mathrm{~kg}$ LiDAR and 7.51t Jet1 fuel), the tested hover time was 1.1 hours (with an average fuel consumption of $0.113 \mathrm{lt} / \mathrm{min})$. 


\subsection{Vibration reduction of the rotor}

The rotor of a helicopter mainly produces low-frequency vibration. In figure 7 , the peak vibration of $1.4 \mathrm{G}, 17.02 \mathrm{~Hz}$ is generated by the main rotor $(1,021.2 \mathrm{r} / \mathrm{min})$. Low-frequency vibration is more difficult to be absorbed by the body or a damper. When a silica gel damper with wider frequency is installed on the gimbal, most of the high and medium frequency vibration can be filtered (Figure 8), but low-frequency vibrations under $0.32 \mathrm{G}, 81.6 \mathrm{~Hz}$ still exist.

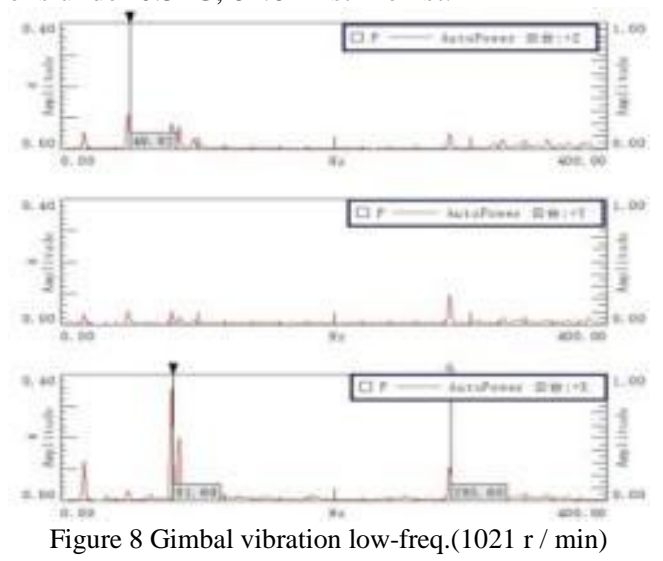

In the absence of atmospheric turbulence, there are two kinds of rotor vibration, the rotating eccentric rotor vibration and the wingtip vortex vibration. Rotating eccentric rotor vibration can be resolved by using paired blades that differ little in weight and center-of-gravity position. Wingtip vortex vibration is formed when the airflow under the wingtip rolls up violently to produce a wingtip vortex, which in turn collides with the rotor wing. This happens when the wingtip is moving at high speed and there exists a differential pressure between the two sides of the rotor wing. In the above test, the three blades of the turbineshaft helicopter have reached a precise balance. Vibration is largely due to the wingtip's straight-cut surface (Figure 9, the example at the top), where vortex occurs early, intensely and directly impacts on the wingtip. The best solution is to sharpen the wingtip and model a sweep back (Figure 9, the lowest example). Thus wingtip vortex is significantly reduced and it occurs in places further towards the rotor shaft, causing less vibration interference on the wingtip. The straight-cut surface of the wingtip will be trimmed, so that the maximum vibration can be further reduced.

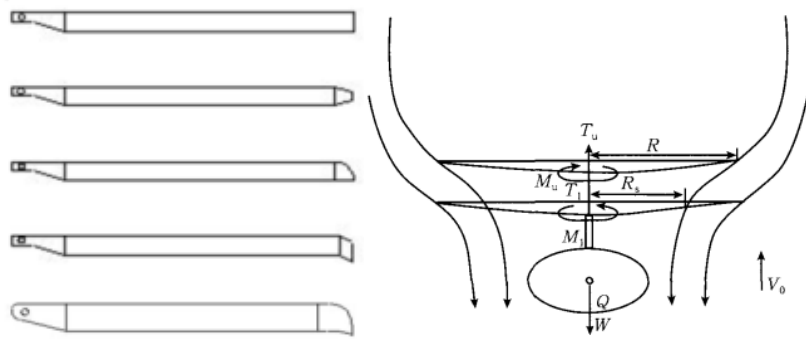

Figure 9 (left) Different wingtip shapes (wingtip vortex decreases, from top to bottom)

Figure 10 (right) Diagram of the flow field of coaxial double rotor (cited from reference [4])

Gears are an essential part of a helicopter and have been developed into many mature types, reaching vibration frequencies between those of an engine and the main rotor. Their vibration is mainly caused by the quality of manufacturing.
This paper does not elaborate on this aspect and focuses more on the aerodynamic layout of the rotor wing.

\subsection{Selecting an aerodynamic layout for the rotor}

4.3.1 Single rotor helicopter has the minimum vibration: An increase in the number of main rotation axes of the helicopter will add to the complexity of the drive system, resulting in greater mechanical drive vibration, compared with a single-rotor helicopter. Moreover, for double-deck rotors (including coaxial and $\mathrm{V}$ rotor), there are airflow disturbances between the two rotors. "For coaxial helicopters in whatever flying state, there are aerodynamic interferences to varying degrees, that is, the upper rotor affects the lower rotor via downwash and the lower rotor affects the upper rotor via flow state changes. Experiments and theoretical studies show that in hovering and low-speed forward flying, the interaction of the rotors makes the downwash velocity of the lower rotor much larger than that of a single rotor; while the downwash velocity of the upper rotor is almost the same or slightly larger than that of a single rotor. The slip flow tube of the upper rotor reduces the radii $(R)$ (Figure 10) at the lower rotor."[4]. Within the range of radii, the lower rotor takes in the downwash flow of the upper rotor, resulting in a more disordered flow state and additional vibration, compared with a single-rotor helicopter. The tail rotor of a single rotor helicopter, also gives rise to vibration. But compared with the main rotor, its small size (1/7 of the diameter of the main rotor) and high rotation speed (5.5 times the main rotor speed) lead to less interference, which can be easily absorbed by a damper. Moreover, the tail rotor is usually installed outside the downwash flow range of the main rotor to avoid mutual interference. Thus single rotor helicopters work in more simple conditions, compared with coaxial or $\mathrm{V}$ rotor helicopters.

4.3.2 Single-rotor helicopters excel in unpowered glide landing: Helicopter accidents are mainly caused by engine failure and mechanical failure in the tail rotor. However, not all helicopters with a power loss or tail rotor failure are bound to crash. "auto-rotate landing" can help them to land safely (in a powerless drop, the main rotor is kept at a proper angle of pitch so that the natural airflow can maintain or even increase the rotation speed. With the momentum gained, one can rapidly increase the pitch angle to slow down the landing before the helicopter crashes to the ground.) While doing heritage mapping, the author did encounter such accidents as an engine failure or small drive link falling off the tail rotor. But thanks to auto-rotation, helicopters usually landed safely without any damage. Rotor inertia plays an important role in the process. Increasing the number of rotors will lead to the scattering of rotational inertia, making "auto-rotate landing" difficult. Among the above mentioned helicopters, the coaxial helicopter has the worst "auto-rotate landing" capability for its auto-rotation is also subject to the mutual interference of the upper and lower rotors. "Due to the presence of aerodynamic interference between the upper and lower rotor, the rotation of a coaxial double rotor is more complex than that of a single rotor in terms of aerodynamic characteristics." [3] The high rotational inertia of the main rotor provides the last guarantee for a helicopter's safety and therefore a single-rotor helicopter is a wise choice to safeguard the system and avoid damage to expensive equipment and sensors.

For single-rotor helicopters, the capability of auto-rotation varies with the number of the blades and three-blade helicopter is the best solution. "Although a three-blade rotor weighs more than a two-blade rotor, it has better spinning performance and 
achieves stable rotation at a lower speed. Therefore, it can improve safety of the auto-rotation. "[3] Besides, it can significantly enhance the total lift of a helicopter, allow the tail pipe to be shortened and reduce whip-end coupled vibration. Based on the above considerations, the authors designed a three-blade single rotor helicopter to be used as a LiDAR carrier.

\section{DESIGN OF A MOTOR-DRIVEN MULTI-ROTOR LIDAR CARRIER}

Although a coaxial double-rotor helicopter is not the most suitable Lidar carrier, its merits should not be ignored. For example, it is small in size, easy to transport and store and easy to control in a vertical drop. These advantages combined with brushless motors give rise to a second Lidar platform - Hexarotor $\mathrm{Y}$ drone (hereinafter referred to as Y6). Y6 and the turbine-shaft helicopter are mutually complementary and they are sure to achieve the best results if used in combination.

\subsection{Reasons for choosing Y6 drone}

According to the number and layout of the rotors, drones may have many different configurations (Figure 11). The authors chose a Y6 for the following reasons.

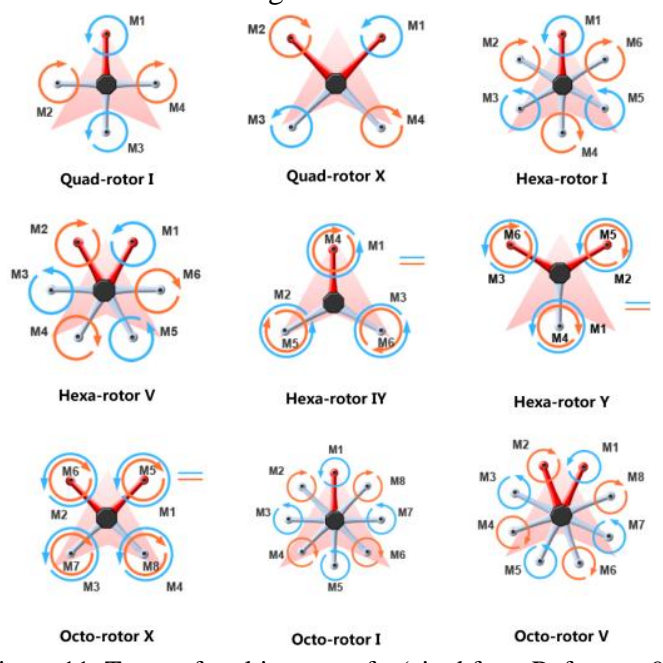

Figure 11 Types of multi-rotorcrafts (cited from Reference 9)

5.1.1 A Y6 is the lightest and smallest and can be folded easily for transportation: Compared with other drones of six or eight motor-mounting arms, the three-arm Y6 significantly reduces its structural weight and increases its load capacity while maintaining the same lift force. Besides, the three mounting arms are easy to fold, which further reduces storage, transportation and other costs. The Y6's small size gives it great flexibility to fly in narrow spaces like treetops and to carry out close-range scanning of relics.

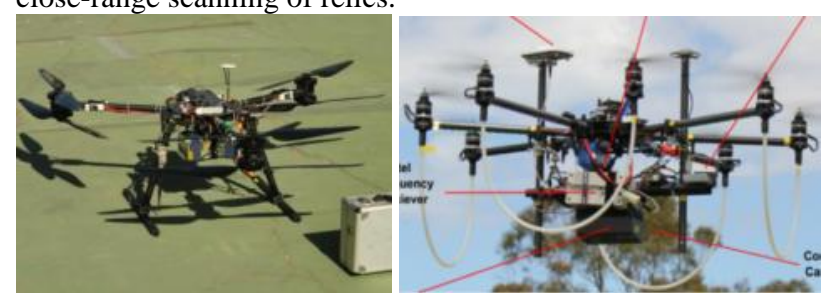

Figure 12 (left) Y6 prototype used for test (equipped with 28-inch blades)

Figure 13 (right) 8-rotor LiDAR system (cited from Reference 8)
To carry a $5-7 \mathrm{~kg}$ LiDAR system, a turbine-shaft helicopter needs to have a total weight of $35-40 \mathrm{~kg}$ and a rotor diameter of more than $2.5 \mathrm{~m}$ (flight duration > 1 hour). If a Y6 drone is used, the total weight of the platform can be reduced to $18-20 \mathrm{~kg}$ and total diameter to less than $1.4 \mathrm{~m}$ when unfolded (flight duration 25-30 minutes). As weight and volume of a Y6 LiDAR platform can be reduced to a minimum, it can ideally be used for smallscale close-range heritage mapping. (Figure 12)

5.1.2 The Y-type structure can use large-diameter rotors and thus has the highest efficiency and longest flight duration: The larger the rotor diameter, the higher the battery's load efficiency (unit: $g / w$ ). Since a drone has limited battery capacity, the key to prolonging its duration is to enhance the power efficiency. Under the same conditions, among all structures, the Y-type structure can use a rotor of maximum diameter. The $12 \mathrm{~kg}$ Y6 drone in our test has interchangeable rotors of two sizes, 28 -inch and 17 -inch respectively. It can hover for about 1 hour $(13 \mathrm{~g} / \mathrm{w})$ when equipped with a 28 -inch rotor, but can only hover 28 minutes with a 17 -inch rotor $(7.5 \mathrm{~g} / \mathrm{w})$. The author of reference [8] finds that duration is significantly inadequate after the multi-rotorcraft is fitted with a LiDAR system. "The main limitation of this platform is the small payload capacity and subsequently the reduced flight time. The electric Oktokopter is only capable of flight times between 3- $5 \mathrm{~min}$."[8] Indeed, the 3-5 min hovering time is of little practical value. Duration is influenced by motor quality, as well as the heavy weight of the structure, small size and low efficiency of the eight-rotor design (Figure 13). If the eightrotor structure is transformed into a Y6, weight will be reduced by $500-800 \mathrm{~g}$ and duration will be increased to $8-10$ minutes. If the rotor diameter is increased further, duration can be further extended to 15-20 minutes, which is then of practical value.

5.1.3 There is no aerodynamic interference among the three arms and in forward flying operation, vibration of the coaxial rotors also decreases: Compared with a coaxial double-rotor helicopter, the blades of the Y6 drone feature small diameter, rigidity and high rotation speed, therefore the vibration interference of the lower rotor caused by the downwash flow is much smaller than that of a coaxial helicopter. It has been proved in actual measurement that in a hover state, the peak vibration acceleration at the center part of the Y-type drone is only $0.13 \mathrm{G}$ (Figure 14), much lower than the peak vibration of the gimbal of a turbine-shaft helicopter after damping.

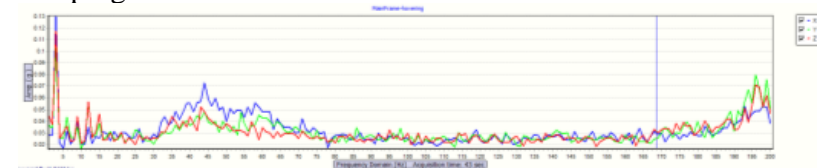

Figure 14 Vibration frequency distribution of Y6 body $(12.4 \mathrm{~kg}$, in hovering state)

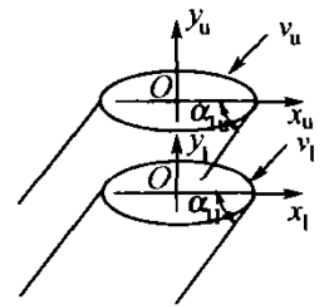

Figure 15 Diagram of the downwash of coaxial rotors in forward flying(reference [5]) 
According to the rotor theory, for a forward-flying coaxial double rotor, the downwash tilts backwards, so the lower rotor can take in more stable incoming air flow, resulting in an enhanced efficiency and stability (Figure 15). This has been verified by Y6 flight tests. In Figure 16, Stage A is the takeoff vertical climbing stage, with the highest power output from the rotor and hence maximum vibration intensity; Stage B is the downwind forward flying stage with a low small airspeed (about $25 \mathrm{~km} / \mathrm{h}$ ) and medium vibration intensity; Stage $\mathrm{C}$ is the upwind flying stage with the highest airspeed (about $50 \mathrm{~km} / \mathrm{h}$ ) and minimum vibration; Stage D is the descending stage, in which the rotor takes in part of its own downwash flow to form a vortex ring, resulting in an increased vibration.

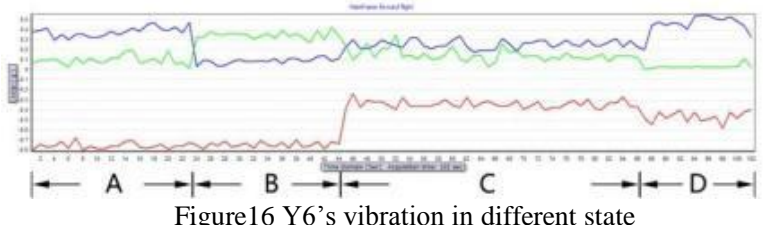

Figure16 Y6's vibration in different state
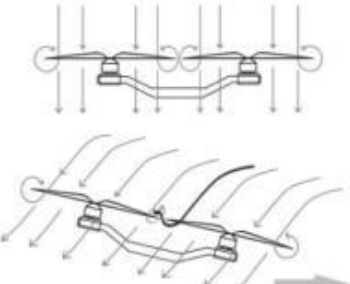

Figure 17 flow interference of single-deck neighboring rotors in forward flying

It has been proved by our tests that when a coaxial double rotor is flying forward or hovering on a windy day, its lower rotor takes in more natural incoming air flow rather than the downwash flow from the upper rotor, resulting in increased efficiency and further reduced vibration. In contrast, for a single-deck drone (e.g. hexa-or octo-rotorcraft), the rotors are arranged too close. In a no-wind hovering state, the air flows do not interfere with each other; but in high-speed forward flying or in windy day, the rear rotor (downwind position) takes in downwash turbulent flow and wingtip vortex of the front rotor (upwind position), resulting in high-intensity arm vibration (Figure 17), which will not only affect the IMU, but also interferes with the autopilot or cause structural body damage. In the known accidents of multi-rotorcrafts, high airspeed flying vibration has been the main cause. Therefore, it is wrong to blindly increase the number and density of the single-deck rotors for the sake of enhancing carrying capacity as this will lead to higher operational risks. Regretfully, few designers, manufacturers and users of multi-rotorcrafts fully understand and give due attention to this problem.

5.1.4 A Y structure blocks the least laser beam: To get a complete scanning of the many surfaces of an ancient building or relic, the laser head should be placed at an optimal forward tilt angle of 45 degrees (Figure 18) so that it not only can scan forward and down, leaving no blind zones on the building façade or top, but also can scan backwards and upwards to obtain data for example, of the roof of a cave. (It is wrong to position the laser head truly horizontally or vertically as this will leave blind zones even with added flight courses.) Under such circumstances, the least rays of a laser beam are blocked by a Y6 drone, ensuring the fullest data set. (Figure 19)..
5.1.5 Compared with other multi-rotorcrafts, a Y6 has an equal or better safety margin: Just as with a single-deck hexadrone, when one rotor fails, a Y6's five other rotors can sustain flying and accomplish emergency landing to protect the full system. In addition, a coaxial double rotor has other advantages: it is capable of a high-speed vertical drop and does not easily fall into a vortex ring and become uncontrollable. In contrast, a single-deck rotor helicopter or a single-deck drone may fall into a vortex ring earlier and, failing to extract itself, is likely to crash in ultra low-altitude operations..

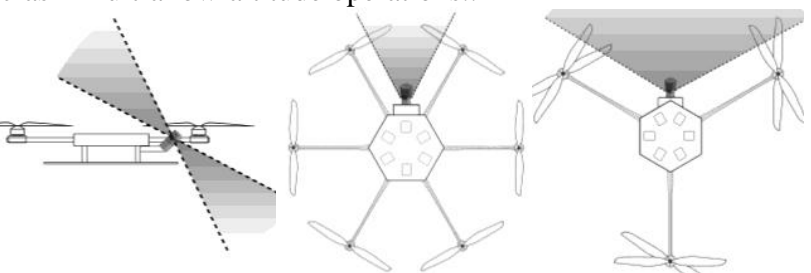

Figure 18 (left) laser head placed at 45 degrees tilting forward Figure 19 (mid\&right) Comparison between two hexa-drones in terms of Blocking of the laser head

5.1.6 With distinct identification features in the front and the back, its movements can be recognized from a distance: To avoid blocking or obscuring details of interest by the body, camera lens or LiDAR, these sensors are usually arranged along the front of the Y-shaped body to form a protruding "nose", very conspicuous and easily recognizable (see Figures 13, 19). In the author's experience, among all multi-rotor types, a Yshape is most easily visible from a distance with the naked eye to identify its direction and movements. In this sense, the Yshape facilitates the monitoring and safety of the drone during flight.

\section{LIDAR DATA ANALYSIS}

To verify the viewpoint in the paper, the authors installed a HDL-32LIDAR system on the unmanned turbine-shaft helicopter to carry out scanning experiments on ancient buildings. A LIDAR sales company also provided scanning data of a riverbank collated by the same scanner installed on a $25 \mathrm{~kg}$ gasoline helicopter. These data were used for comparative analysis of the two platforms. The point-cloud error of ancient buildings is only $2-3 \mathrm{~cm}$, leaving roof ornaments highly distinct(Fig.20); while the error of the bank is so big that even in its best section, the point-cloud discrete diameter of lamp posts exceeds one meter(Fig.21).

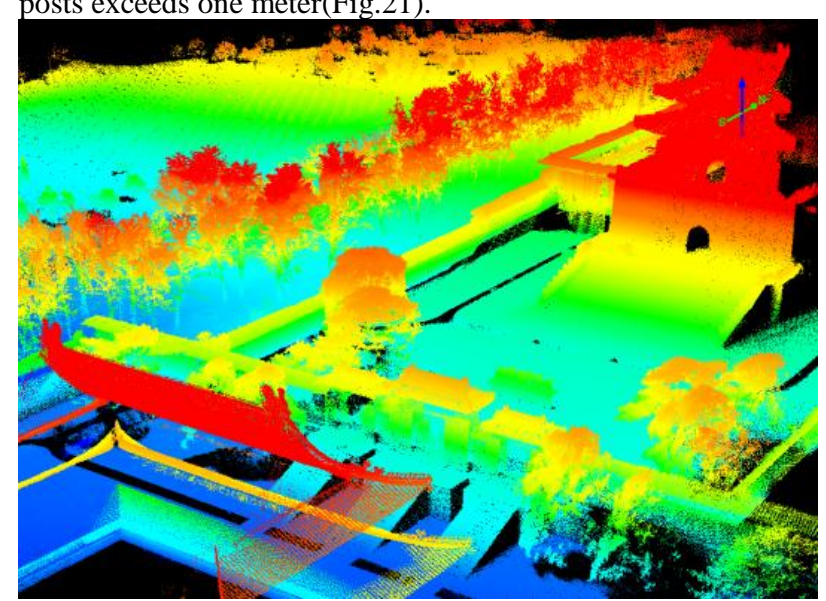

Figure 20 scanning data of an ancient building complex (partial) 


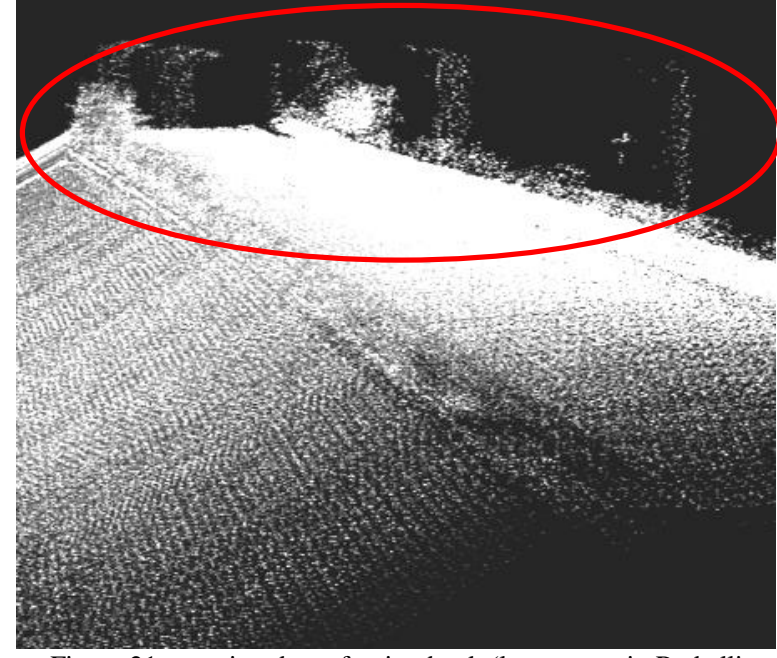

Figure 21 scanning data of a riverbank (lamp posts in Red ellipse)

By amplifying and comparing the data recorded by INS on two different helicopters, we can safely conclude that vibration difference gives rise to difference in scanning accuracy. In Figure 22, INS gesture data record a $1.5 \mathrm{~Hz}, 1$-degree lowfrequency vibration on the two horizontal axes (Omiga, Phi) of the gasoline helicopter. If we amplify the graph, we can see that this low-frequency vibration is loaded with another $24 \mathrm{~Hz}, 0.15$ degree vibration (Fig. 23). In comparison, a turbine-shaft helicopter produces only $16 \mathrm{~Hz}, 0.1$-degree vibration on the two horizontal axes (Fig. 24). On vertical axis (Kappa), the gasoline helicopter produces a $24 \mathrm{~Hz}, 0.2$-degree vibration, while a turbine-shaft helicopter produces only $16 \mathrm{~Hz}, 0.02$-degree vibration(Fig.25).

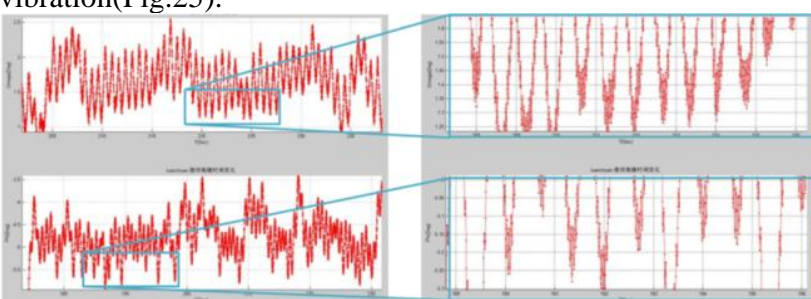

Figure 22 (left) INS data on Omiga and Phi axis of gasoline helicopter Figure 23 (right) amplified data on Omiga and Phi axis of gasoline helicopter

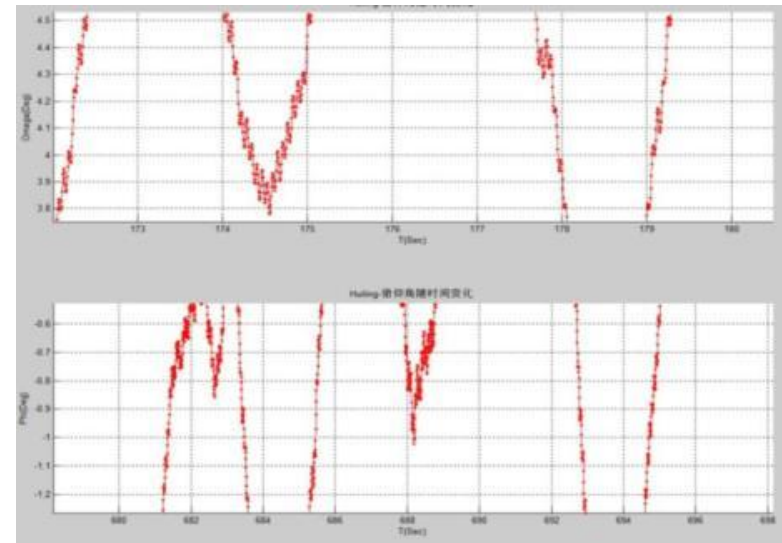

Figure 24 INS data on Omiga and Phi axis of turbine-shaft helicopter

INS has limited measurement capacity. IMU of the LIDAR system features a $200 \mathrm{~Hz}$ sampling rate and a \pm 0.0025 degree drift in angular accuracy. Therefore, it is impossible for a gasoline helicopter platform with high vibration intensity and wide distribution of vibration frequency to have only two vibration frequencies. As IMU cannot measure and filter all vibration disturbances, vibration disturbances become the main cause of the discrete point clouds of lamp posts.

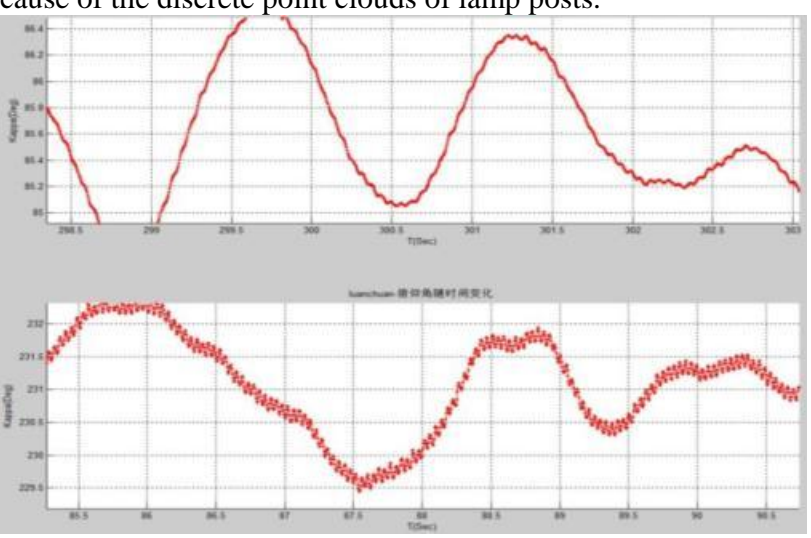

Figure 25 comparison of Kappa data (up: turbine-heli; down: gasolineheli)

In Figure 24 and 25, gentle curve fluctuations with frequencies below $0.5 \mathrm{~Hz}$ are caused by attitude adjustment of the aircraft. These low-frequency fluctuations can be accurately measured by POS system and will not significantly affect data accuracy.

\section{CONCLUSION AND OUTLOOK}

Due to its limited angular measurement capability, IMU is used to measure tilt and swing rather than high-frequency vibration of the moving platform. Therefore, when we use an unmanned aircraft to obtain high-precision laser scan data of architectural heritages and relics, we need to take into account factors like power type and fuselage design so as to minimize vibration of the aircraft itself. Other means such as vibration reduction and smoothing should also be adopted. Thus by integrating multidisciplinary advantages, we can get double result with half effort.

No platform is a cure-all. Even in mere heritage scanning, large and small rotor UAVs with their respective advantages are both needed to achieve maximum results. In the mapping of ancient architectures, due to its large size and inadequate flexibility, a turbine-shaft helicopter can hardly reach and scan the wooden structure under the eave. Therefore, the authors will continue to do scanning experiments with battery powered drone LIDAR.

UAV is undergoing constant improvement. The emergence of some new safety equipment such as "emergency parachute" of rotor-wing aircrafts might undermine some conclusions of this paper. But new equipment is like a double-edged sword. For example, a parachute can enhance the safety of unpowered emergency landing of a multi-rotor aircraft, but it will also significantly shorten battery time. Therefore, the conclusions of this paper will remain useful for quite some time in the field of heritage mapping.

Low-altitude laser scanning platform will soon be widely applied in the mapping of ancient architectures. With increasing scanning accuracy, LIDAR's centimeter-level scanning accuracy is close to the terrestrial laser scanning accuracy, which can meet the general requirements of ancient architecture mapping. Besides, time required for LIDAR scanning is only $1 /$ $50 \sim 1 / 100$ that of terrestrial scanning. So the outlook of its application is optimistic. The main blades used in this test has a straight-cut wing tip. Vortex generated by straight wingtip causes a major $16 \mathrm{~Hz}$ vibration disturbance, so authors are now replacing the rotor. We are confident that in subsequent 
experiments with lower vibration disturbance and better scanning mode, we can expect to obtain data with an error of close to $1 \mathrm{~cm}$.

\section{ACKNOWLEDGEMENTS (OPTIONAL)}

The project is funded by National Nature Science Foundation of China (project number: 51478298).

\section{REFERENCES}

Ma Hongchao and Wu Jianwei, "Analysis of Positioning Errors Caused by Platform Vibration of Airborne LiDAR System", 2012 8th IEEE International Symposium on Instrumentation and Control Technology(ISICT), P257-261,11-13 July 2012

C.F.Periu, A.Mohsenimanesh, C.Laguë and N.B.McLaughlin, Isolation of Vibrations Transmitted to a LIDAR Sensor Mounted on an Agricultural Vehicle to Improve Obstacle Detection, CANADIAN BIOSYSTEMS ENGINEERING, P2.33-2.42, Volume 55, 2013

JI Leqiang, ZHU Qinghua, LI Jianbo, Experimental investigation on aerodynamic characteristics of auto rotating coaxial twin - rotor and single rotor, Journal of Experiments in Fluid Mechanics, Vo1. 27. No. 5, P7-10, 0ct. 2013

Chen Ming, Technology Characteristic and Development of Coaxial Rotor Helicopter, Aeronautical Manufacturing Technology, 2009 No.5, P26-31

Chen Ming and Hu Jizhong and Cao Yihu, Rigid-wake analysis of coaxial rotor aerodynamics in forward flight, Journal of Beijing University of Aeronautics and Astronautics, Vol.30, No.1,P74-78, January 2004

HDL-32E Datasheet, Velodyne Acoustics, Inc. www. velodynelidar.com

FU Ji-bo, MA Jing, YAO Jian-jun, Coupling vibration study on the elastic supported inertia navigation system, STRUCTURE \& ENVIRONMENT ENGINEERING, Vol 32 No.2, P46-51, Jun.2005

Luke Wallace, Arko Lucieer, Christopher Watson and Darren Turner, Development of a UAV-LiDAR System with Application to Forest Inventory, Remote Sens. 2012, 4, 15191543; doi:10.3390/rs4061519

WKM QUICK START GUIDE V1.12, DJI Innovations. January 2, 2014 Revision, www.dji.com

ZHAO Lijian, CHENG Xinwen, LI Yingcheng, CHEN Gang, NIE Xiaobo, Accuracy Check and Analysis of LIDAR Elevation Data, GEOSPATIAL INFORMATION, Vol.7 No.1, P58-60, Feb. 2009 